\title{
Near-field photoluminescence spectroscopy of localized states in InGaAsN alloys.
}

\section{A. M. Mintairov ${ }^{1,2}$, P. A. Blagnov ${ }^{2}$, T. Kosel $^{1}$, J. L. Merz ${ }^{1}$, V. M. Ustinov ${ }^{2}$, A. S. Vlasov ${ }^{1}$, R.} E. $\operatorname{Cook}^{3}$.

${ }^{1}$ Electrical Engineering Department, University of Notre Dame, Notre Dame, IN 46556

${ }^{2}$ Ioffe Physical-Technical Institute RAS, Polytechnicheskaya 26, St. Petersburg, Russia

${ }^{3}$ Argonne National Laboratory, Argonne, IL 60439.

\begin{abstract}
We used near-field magneto-photoluminescence scanning microscopy to study structural and optical properties of quantum-dot-like compositional fluctuations in GaAsN and InGaAsN alloys. We show that these fluctuations manifest themselves by the appearance of narrow emission lines (halfwidth $0.5-2 \mathrm{meV}$ ) at temperatures below $70 \mathrm{~K}$. We estimated the size, density, and nitrogen excess of individual compositional fluctuations (clusters), revealing phaseseparation effects in the distribution of nitrogen in GaAsN and InGaAsN. We found a dramatic difference in the Zeeman splitting of cluster lines between GaAsN and InGaAsN, indicating a strong effect of In on the exciton $g$-factor.
\end{abstract}

\section{INTRODUCTION}

$\mathrm{In}_{x} \mathrm{Ga}_{1-x} \mathrm{As}_{1-y} \mathrm{~N}_{y}(x \sim 0-0.08, y \sim 0-0.05)$ alloys have recently attracted considerable attention as promising materials for laser diodes in the $1.3-1.5 \mu \mathrm{m}$ range as well as more efficient solar cells. These applications exploit their unusual electronic property - a "giant bowing" parameter $(b \sim 20 \mathrm{eV})$, which arises from the large electronegativity and small size of the nitrogen $[1,2]$. In our recent study [3] we used temperature-dependent near-field magneto-photoluminescence spectra to observe the localization of excitons on quantum-dot-like (QD-like) compositional fluctuations of $\operatorname{In}_{0.08} \mathrm{Ga}_{0.92} \mathrm{As}_{0.97} \mathrm{~N}_{0.03}$. Localization is driven by the giant bowing parameter of these alloys and manifests itself by the appearance of ultranarrow lines (halfwidth $<1 \mathrm{meV}$ ) at temperatures below $70 \mathrm{~K}$. We used near-field magneto-photoluminescence spectra for the estimation of the size, density, and nitrogen excess of individual compositional fluctuations (clusters) in InGaAsN, thus revealing random versus phase-separation effects in the distribution of nitrogen.

In the present paper we extend these magneto-optical measurements together with transmission electron microscope measurements to include GaAsN alloys with the same nitrogen content, allowing us to study the effect of In incorporation on the optical and structural properties of QD-like compositional fluctuation. We observed a similarity in size, $\mathrm{N}$ content and density of QD-like clusters in GaAsN and InGaAsN. However, Zeeman splitting was not observed for cluster emission lines in GaAsN, indicating a strong effect of In on the exciton $g$-factor in InGaAsN alloys.

\section{EXPERIMENT}

The $\mathrm{GaAs}_{1-\mathrm{y}} \mathrm{N}_{\mathrm{y}}$ and $\mathrm{In}_{x} \mathrm{Ga}_{1-x} \mathrm{As}_{1-y} \mathrm{~N}_{y}(\mathrm{x}=0.08, \mathrm{y}=0.03)$ samples used in this study were grown by solid source molecular beam epitaxy on (001) semi-insulating GaAs substrates at $\mathrm{T}=450^{\circ} \mathrm{C}$. The layers with thickness of $0.12 \mu \mathrm{m}$ were sandwiched between AlGaAs layers of 
thickness $60 \mathrm{~nm}$ (bottom) and $14 \mathrm{~nm}$ (top). The InGaAsN layer consisted of a short period $\mathrm{GaAs}_{0.966} \mathrm{~N}_{0.034} /$ InAs superlattice with individual layer thicknesses of 2.82 and $0.25 \mathrm{~nm}$, respectively. The structure was capped by a $20 \mathrm{~nm} \mathrm{GaAs}$ layer and annealed at $710^{\circ} \mathrm{C}$ for $10 \mathrm{~min}$. The far-field PL spectrum of the layers at $5 \mathrm{~K}$ consisted of a single broad band (halfwidth $\gamma \sim 20$ $\mathrm{meV}$ ) with peak energy 1.11 and $1.09 \mathrm{eV}$, similar to results reported in the literature [4].

Near-field photoluminescence (NPL) spectra with spatial and spectral resolution of 300 $\mathrm{nm}$ and $0.4 \mathrm{~nm}(0.5 \mathrm{meV})$, respectively, were taken in collection-illumination mode using uncoated tapered fiber tips. The spectra were excited by $20 \mu \mathrm{W}$ of Ar ion laser excitation at $514.5 \mathrm{~nm}$, measured in the temperature range 5-300 K and magnetic field strengths 0-10 T.

A cross-sectional specimen was prepared for transmission electron microscopy (TEM) by wedge polishing and Ar ion milling with liquid nitrogen cooling; the final accelerating energy was $500 \mathrm{~V}$. TEM observations were done at $200 \mathrm{kV}$ in a Philips CM30 microscope.

\section{RESULTS AND DISCUSSION}

$i$ - Near-field spectra. In Fig. $1 \mathrm{a}, \mathrm{b}$ we present NPL spectra of our samples taken at $5 \mathrm{~K}$. We see that at $\mathrm{T}=5 \mathrm{~K}$ the spectra reveal structure, consisting of the series of multiple peaks

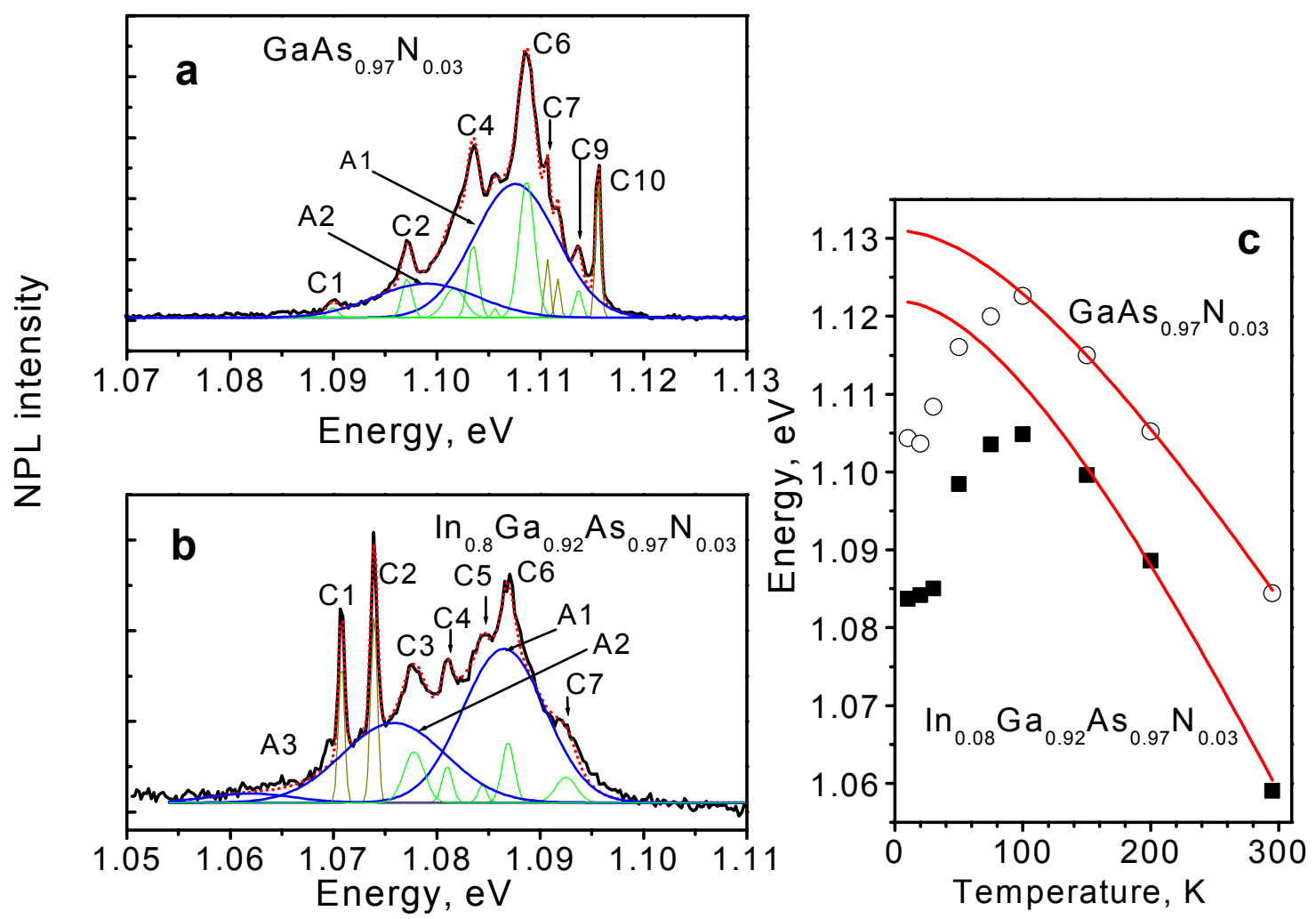

Figure 1. Experimental (thick solid) and multiple Gaussian peak modeling (dotted whole spectra and thin solid - constituents) of $5 \mathrm{~K}$ near-field PL spectra of $\mathrm{GaAs}_{0.97} \mathrm{~N}_{0.03}$ (a) and $\mathrm{In}_{0.08} \mathrm{Ga}_{0.92} \mathrm{As}_{0.97} \mathrm{~N}_{0.03}$ (b). (c) shows the temperature dependence of the maximum of the band A1 (solid squares - $\operatorname{In}_{0.08} \mathrm{Ga}_{0.92} \mathrm{As}_{0.97} \mathrm{~N}_{0.03}$, open circles - $\mathrm{GaAs}_{0.97} \mathrm{~N}_{0.03}$ ) measured in the spectra and calculated using Varshni's expression [5] (solid curve). 
centered at $1.108 \mathrm{eV}$ in GaAsN and at $1.087 \mathrm{eV}$ in InGaAsN. For both alloys a Gaussian contour decomposition resolves up to ten narrow lines $(\gamma=0.5-2 \mathrm{meV})$, denoted C1-C10, superimposed on the broader bands $(\gamma \sim 8-10 \mathrm{meV})$, denoted A1-A3. We observed [3] that the intensities of the $\mathrm{C}$-lines decrease dramatically with increasing temperature, disappearing at $\sim 70 \mathrm{~K}$. In Fig. 1, $\mathrm{c}$ the temperature dependence of the energy maximum of the A1 band in GaAsN and InGaAsN is shown. We can see that this dependence is similar in both alloys. For T $>100 \mathrm{~K}$ the shift of the A1 band energy follows the behavior typical for band gap emission of a bulk semiconductor: a decrease of the emission energy with increasing temperature due to thermal expansion, which is well described by the Varshni model [3,5]. For lower temperatures, however, the A1 band shows an inverted behavior, which indicates onset of the carrier localization. We attribute the Abands to the emission of excitons localized on composition fluctuations having spatial extent $2 r$, much greater than the exciton radius $\left(r_{\text {exc }}\right)$ which is $\sim 10 \mathrm{~nm}$ (weak localization regime), and we attribute the C-lines to emission of excitons localized on QD-like clusters having $r \leq r_{\text {exc }}$ (strong localization regime). The difference between Varshni's model predictions and the observed energy positions of the emission lines for $\mathrm{T}<150$ (Fig. 1, c) gives the exciton localization energy, which is equal to $10-60 \mathrm{meV}$ for $\mathrm{T}=5 \mathrm{~K}$.

ii - Magneto NPL spectra. We used magneto-photoluminescence measurements to estimate the size of the composition fluctuations. In Fig. 2, a and b we present the magnetic field dependence of the C-lines in the GaAsN and InGaAsN samples. We observed Zeeman splitting of the C-lines in InGaAsN (subscript $h$ and 1 denote high and low energy components in Fig2, b). The splitting energy has values of $0.6 \mathrm{meV}$ for $4 \mathrm{~T}$ and $1.7 \mathrm{meV}$ for $10 \mathrm{~T}$, which is typical for GaAs [6] and InGaAs [7] QDs and corresponds to an exciton $g$-factor close to two. However, we did not observe Zeeman splitting of the C-lines in GaAsN (Fig.2,a); i.e. the g-factor is zero. Since the
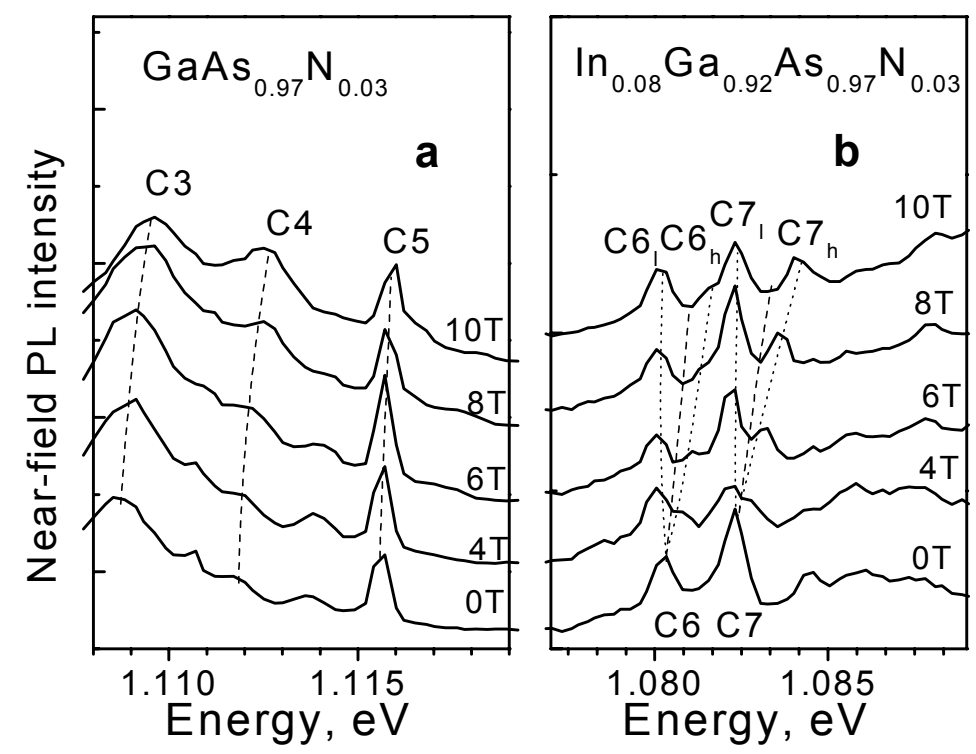

Figure 2. 5K near-field PL spectra of GaAsN (a) and InGaAsN (b) at magnetic field strengths $\mathrm{B}=0,4,6,8,10 \mathrm{~T}$. 


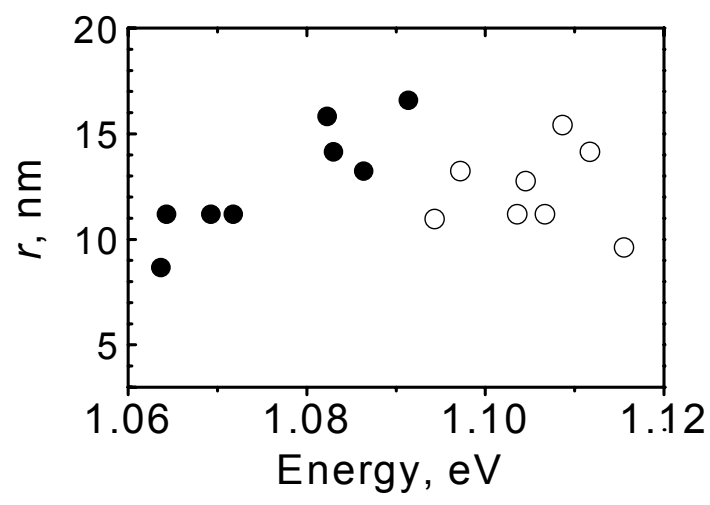

Figure. 3. Cluster radius calculated from the values of the diamagnetic coefficient $\beta$ [3] (open circles - GaAsN, solid circles - InGaAsN) versus emission energy of C-lines.

exciton $g$ factor in bulk GaN is zero for a magnetic field parallel to the $c$-axis [8], this observation suggest a very high $\mathrm{N}$ content $(\Delta y>>y)$ of the clusters.

In contrast to the Zeeman splitting results, we observed a clear diamagnetic shift for the $\mathrm{C}$-lines in both GaAsN and InGaAsN alloys. We measured the value of the diamagnetic coefficient to be $\beta=3-12 \mu \mathrm{eV} / \mathrm{T}^{2}$. The cluster radius for different emission energies deduced from $\beta$ [3] is presented in Fig. 3. We can see that in both samples it has a value $12 \pm 5 \mathrm{~nm}$.

It should be noted that in GaAsN for energy $>1.09 \mathrm{eV}$ some $\mathrm{C}$-lines have a low energy (paramagnetic) shift in the magnetic field, which reaches a value of $0.4 \mathrm{meV}$ at $10 \mathrm{~T}$. The origin of such shift is not clear at this time.

We also find that the A-bands have a diamagnetic coefficient of $30-45 \mu \mathrm{eV} / \mathrm{T}^{2}$, corresponding to $2 r \sim 50-60 \mathrm{~nm}$, which is consistent with the weak localization regime.

Using a calculation of the ground state energy of a particle in a spherical confining region [9] having a radius of $12 \mathrm{~nm}$ and localization energy of $40 \mathrm{meV}$, we calculated the value of the confining potential to be $80 \mathrm{meV}$ [3]. This gives the average value of the compositional fluctuation in the cluster to be $\Delta y \sim 0.5 \%$.

For $r \sim 12 \mathrm{~nm}$ ( 80000 bonds) and $\Delta y \sim 0.5 \%$ Stirling's formula (random alloy) gives the probability of cluster formation to be as low as $10^{-15}$. This corresponds to a density of the clusters of $2 * 10^{-8} \mu \mathrm{m}^{-3}$ which is too low to describe the observation of several emission cluster lines in our experiments (see below) and suggests that the formation of the clusters in our samples is spontaneous.

iii - Near-field PL imaging. The lateral distribution of the composition fluctuations was investigated using near-field PL scanning experiments. These measurements also allow us to estimate the density of the QD-like clusters in our samples to be $100 \mu \mathrm{m}^{-3}$, which is ten orders of magnitude higher than that predicted for random alloy.

Fig. 4 presents selected monochromatic NPL intensity images (scans a-e) of the $2 \times 2 \mu \mathrm{m}$ area of the GaAsN sample taken with $\mathrm{x} / \mathrm{y}$ steps of $0.2 \mu \mathrm{m}$. The NPL spectra, measured at positions having maximum intensity in scans a-e are also presented. Simultaneous analysis of the images and the spectra shows that the spatial resolution of our experiments is $300 \mathrm{~nm}$. This is clearly seen in images in the upper left section in scan c and in scan e. The higher spatial scale of the images (up to $600 \mathrm{~nm}$ ) as well as their elongated character seen in the scans a-d are due to 


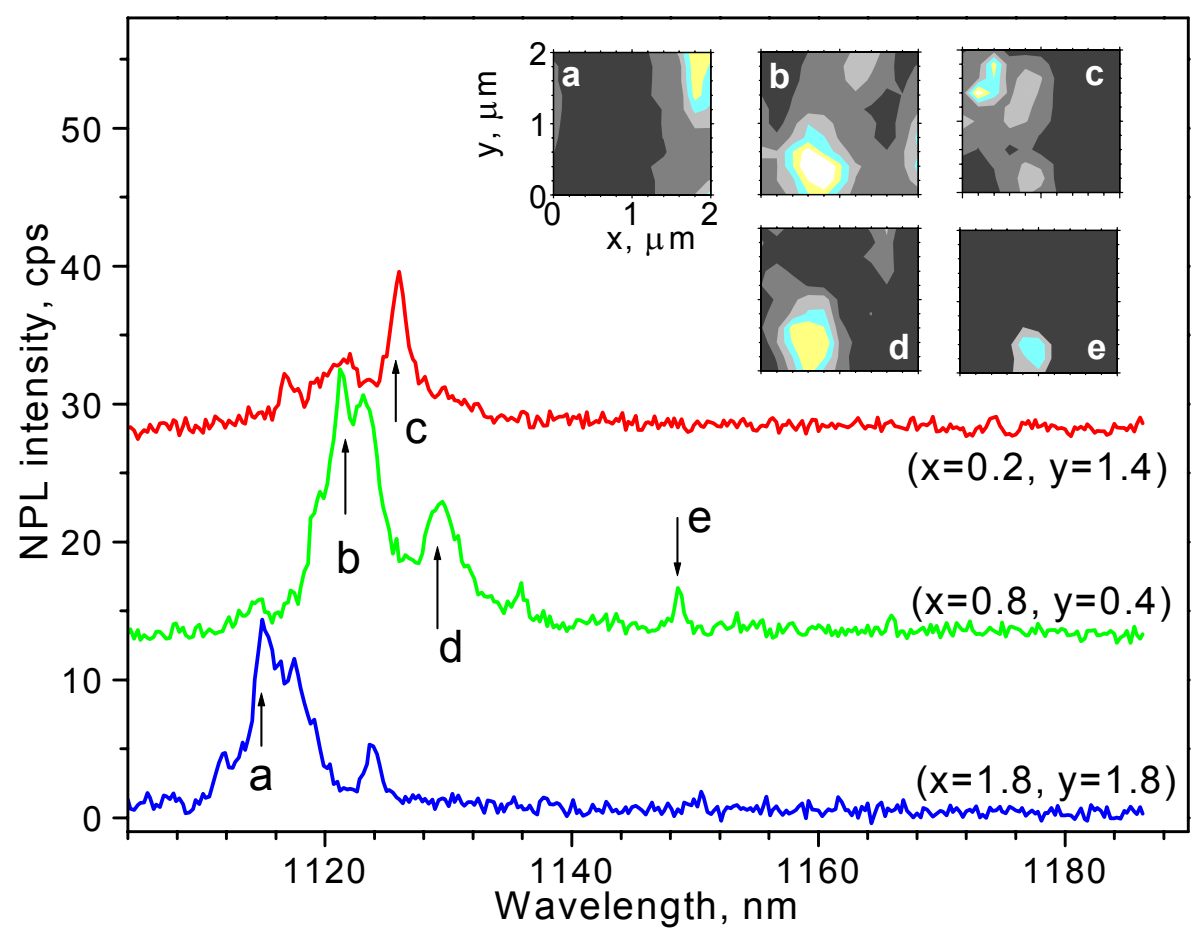

Figure 4. Monochromatic NPL intensity scans (a-e) of a $2 \times 2 \mathrm{~mm}$ area of $\mathrm{GaAs}_{0.97} \mathrm{~N}_{0.03}$ together with NPL spectra (arrows show detection wavelength in corresponding scans) taken at positions $(\mathrm{x}, \mathrm{y}), \mu \mathrm{m}:(0.2,1.4),(0.8,0.4)$ and $(1.8,1.4)$ with maximum intensity.

the overlapping of different lines. All scans presented (except scan e) show locations for which at least five clusters are emitting at wavelengths within $1 \mathrm{~nm}$ of the detection wavelength. Our measurements reveal a strong inhomogeneity of the GaAsN layer on a length scale of $1 \mu \mathrm{m}$. Indeed, in the scan $\mathrm{c}$ the emission of the upper left $1 \mathrm{x} 1 \mu \mathrm{m}$ section of the scan area is dominated by five clusters laterally separated by nearly $0.4 \mu \mathrm{m}$. The emission wavelengths of these clusters occupy a very small spectral range of $\sim 2 \mathrm{~nm}$, indicating small difference (narrow distribution) in the cluster size and $\mathrm{N}$ content. In contrast, in the lower left section the cluster separation is much smaller $(<0.2 \mathrm{~nm})$ but their wavelengths occupy spectral range $\sim 30 \mathrm{~nm}$ (scans b-e), indicating a broad distribution of the sizes and N content. Similar long-scale inhomogenity was observed in the InGaAsN sample.

$i v-T E M$ study. Additional evidence of cluster formation in our samples was obtained using TEM measurements. Fig. 5, a-c shows bright-field TEM images of GaAsN and InGaAsN specimens. All images show diffraction contrast variations which we attribute to composition fluctuations. In the $\mathrm{g}=004$ image of $\operatorname{InGaAsN}$ (Fig. 5,a) this appears as a merging of the several superlattice layers and has lateral size $10-30 \mathrm{~nm}$, which is in a good agreement with our estimation of the lateral cluster size from near-field magneto-PL spectra. We found that this contrast is observable only at exactly the Bragg angle $(\mathrm{s}=0)$. Fig.5,b shows a 2-20 image of the same specimen. The contrast variation in this case has a more vertical appearance, similar to that observed in conventional III-V alloys [10]. It has the same lateral size as seen with 004 contrast. 
The contrast extends below the InGaAsN layer, where a smaller amount of $\mathrm{N}$ is present. Fig.5, c is a 2-20 image of GaAsN, showing similar contrast to Fig.5, b.
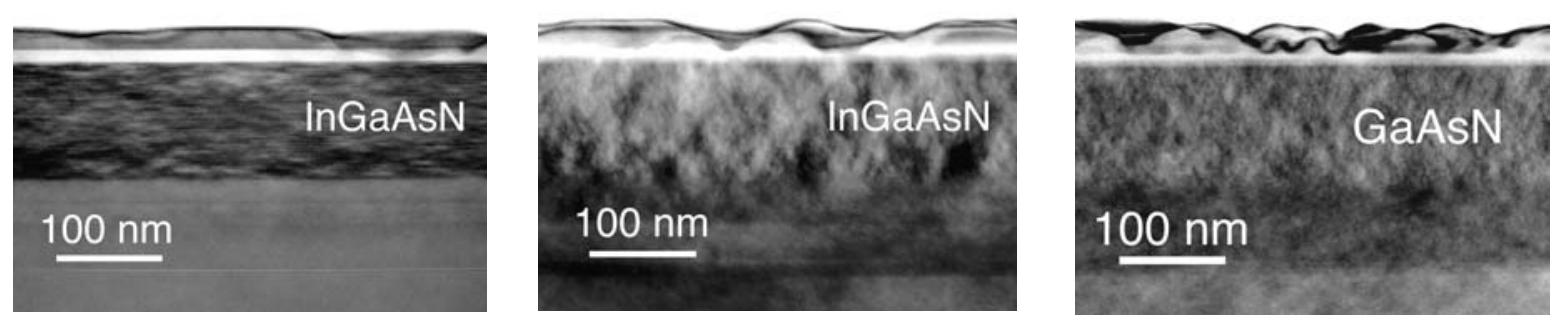

Figure 5. Bright-field TEM images of $\operatorname{In}_{0.08} \mathrm{Ga}_{0.92} \mathrm{As}_{0.97} \mathrm{~N}_{0.03}$ (a- 004, b- 2-20) and $\mathrm{GaAs}_{0.97} \mathrm{~N}_{0.03}(\mathrm{c}-2-20)$ structure.

\section{CONCLUSION}

Using near-field magneto-photoluminescence scanning microscopy and transmission electron microscopy we observed quantum-dot-like compositional fluctuations in GaAsN and InGaAsN alloys. These fluctuations manifest themselves by the appearance of narrow lines (halfwidth $0.5-2 \mathrm{meV}$ ) at temperatures below $70 \mathrm{~K}$. We used near-field magneto-optical scanning microscopy for the estimation of the size, density, and nitrogen excess of individual compositional fluctuations (clusters) and reveal phase-separation effects in the distribution of nitrogen. A strong effect of indium on the exciton g-factor was observed.

\section{ACKNOWLEDGEMENTS}

The authors wish to acknowledge the W. M. Keck Foundation and the NATO Science for Peace Program (Grant SFP-972484) for support of this research. The TEM work at Argonne National Laboratory was supported by the U. S. Department of Energy, Office of Science, under Contract W-31-109-Eng-38.

\section{REFERENCES}

1. S. Sakai, Y. Ueta and Y. Terauchi, Jpn. J. Appl. Phys. 32, p. 4413 (1993).

2. S.-H. Wei and A. Zunger, Phys. Rev. Lett. 76, 664 (1996).

3. A. M. Mintairov, P. A. Blagnov, T. Kosel, J. L. Merz, V. M. Ustinov, A. S. Vlasov, R. E. Cook, Phys. Rev. Lett. (in press)

4. E. D. Jones, A. A. Allerman, S. R. Kurz, N. A. Modine, K. K. Bajaj, S. W. Tozer and X. Wei, Phys. Rev. B 62, 7144 (2000).

5. Y. P. Varshni, Physica 34, 149 (1967).

6. U. Bockelman, W. Heller and A. Abstreiter, Phys. Rev. B 55, 4469 (1997)

7. Y. Toda, S. Shinomori, K. Suzuki and Y. Arakawa, Appl. Phys. Lett. 73, 517 (1998).

8. R. Stepniewski, M. Potemski, A. Wysmolek, K. Pakula, J. M. Baranowski, J. Lusakowski, I. Grzegory, S. Porowski, G. Martinez and P. Wyder, Phys. Rev. B 60, 4438 (1999).

9. D. B. Tran Thoai, Y. Z. Hu and S. W. Koch Phys. Rev. B 42, 11261, (1990). 
10. A. Zunger and S.Mahajan, in Handbook on semiconductors, v.3, Elsevier Amsterdam, (1994) 